\title{
Role of Structural Heterogeneities on Segmental Orientation in Deformed Chains: Application to Alternating Copolymers
}

\author{
Ivet Bahar," Burak Erman, and Turkan Haliloglu \\ Polymer Research Center and School of Engineering, Bogazici University, and TUBITAK \\ Advanced Polymeric Materials Research Center, Bebek 80815, Istanbul, Turkey
}

Received April 14, 1993; Revised Manuscript Received December 9, $1993^{\circ}$

\begin{abstract}
Effects of intrinsic structural and conformational properties on segmental orientation in uniaxially deformed copolymers are considered. Depedence of segmental orientation on equilibrium values of bond angles, torsional states, and probability distribution of rotameric states is studied. Calculations are carried out for chains with independent as well as pairwise interdependent rotameric states for neighboring bonds using the matrix generation technique of rotational isomeric state formalism. Results invite attention to the importance of specific energy and geometry parameters in prescribing the level of molecular orientation in the two different components $A$ and $B$ of $A B$ type copolymers. Results are interpreted with reference to polarized Fourier transform infrared spectroscopy measurements in which the orientation of transition moment vectors is detected. The consequences of certain assumptions in data interpretation such as the choice of cylindrically symmetric reference axes along the chain contour are pointed out. The orientations of vectors along the backbone exhibit strong nonlinear dependence on the conformational characteristics of the component A or $B$ to which they are appended. Thus, the bond vectors of the two monomeric units may exhibit quite distinct orientations, arising only from slight perturbation in bond angles of one of the units. Vectors perpendicular to chain backbone were less sensitive to monomeric structure. Calculations carried out independently by Monte Carlo simulations showed that this method yields an adequate qualitative description of the orientational behavior of chain segments while precise quantitative determination requires the use of the exact matrix generation technique.
\end{abstract}

\section{Introduction}

With the advance of experimental methods for measuring molecular orientation, such as polarized Fourier transform infrared ${ }^{1}$ (FTIR) and deuterium nuclear magnetic resonance $\mathrm{e}^{2,3}\left({ }^{2} \mathrm{H}-\mathrm{NMR}\right)$ spectroscopy, more attention is being invited to the role of structural and conformational characteristics of polymer chains in prescribing the observed behavior. ${ }^{4}$ The analysis of segmental orientation with these techniques, in networks and bulk polymers above the glass transition temperature, leads to information that is commensurate, in precision and in detail, with results of calculations based on the rotational isomeric state (RIS) model of chain statistics. ${ }^{5}$ Recent comparison of FTIR measurements on well-defined model poly(dimethylsiloxane) (PDMS) networks with RIS calculations, ${ }^{1}$ for example, shows satisfactory agreement between experiment and theory. These measurements and calculations are also in reasonable accord with the ${ }^{2} \mathrm{H}-\mathrm{NMR}$ data of Deloche et al.,6,7 on similar PDMS networks. Further progress in FTIR spectroscopy by the introduction of modulation techniques ${ }^{8}$ renders this experimental field even more challenging. The careful analysis of segmental orientation in elastomeric networks, in particular, leads to indispensable information in understanding the physics of dense polymeric media.

Both the FTIR and the ${ }^{2} \mathrm{H}-\mathrm{NMR}$ techniques directly measure the orientation of specific labels on a chain relative to a laboratory-fixed axis. The orientation is suitably induced by stretching the specimen uniaxially. The sample may be an elastomeric network or an un-cross-linked sample. In the case of a network, segmental orientation at equilibrium may be obtained. Furthermore, the system may be swollen with a suitable diluent to eliminate local intermolecular orientational correlations. ${ }^{9}$ In this way, single chain configurational contributions to segmental orientation may be measured and compared with predic-

- Abstract published in Advance ACS Abstracts, February 15, 1994.

$0024-9297 / 94 / 2227-1703 \$ 04.50 / 0$ tions of statistical theories. In un-cross-linked samples, orientation is induced by deforming the sample macroscopically at a rate sufficiently high to prevent relaxation.

There has been noteworthy progress in this field during recent years, ${ }^{10-16}$ especially in the field of engineering polymers and blends. A recent infrared study ${ }^{13}$ of orientation and chain relaxation of amorphous homopolymers or miscible polymer blends stretched above their glass-transition temperature indicates that local forces between neighboring segments play a major role in the observed behavior. Local forces may be either of intrachain or interchain origin. Intrachain forces arise from the intrinsic chain stiffness or reduced flexibility along specific directions, depending on the particular chain geometry and conformational energetics, whereas interchain interactions impose spatial restrictions on the reorientation motion of the chain segments, becoming stronger in denser media. An interesting feature which emerges from experimental studies is, however, the different levels of orientation observed for the components of a given blend. This behavior is valid both in compatible amorphous blends, in which favorable intermolecular interactions might induce some orientational coupling between the components, and in blends where interchain interactions are asserted to be nonexistent. ${ }^{13}$ Examples are the blends of polystyrene-poly(xylene ether), ${ }^{17}$ polystyrene-poly(vinyl methyl ether), ${ }^{18}$ poly (methyl methacrylate)-co-poly(styrene-co-acrylonitrile). ${ }^{13}$ The fact that the different components exhibit different orientations irrespective of intermolecular coupling, if any, suggests that the intrinsic structural and conformational properties of the individual chains might be predominant in controlling the observed orientations. This argument is further strengthened by the experimental observation of different orientations in the two different structural units of polystyrene-poly(vinyl methyl ether) copolymer chains.8

The specific purpose of the present communication is to emphasize the importance of the role of intrinsic structural and conformational characteristics of the single polymer chain in segmental orientation. For this purpose, 


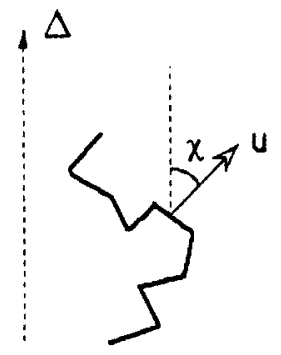

(a)

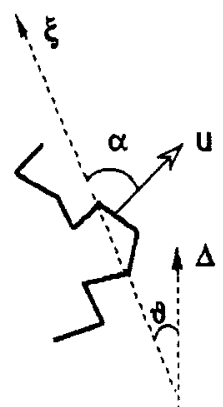

(b)

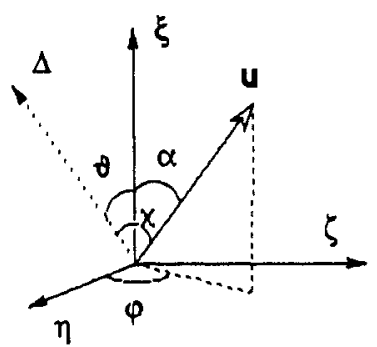

(c)

Figure 1. (a) Orientation of the investigated vector $u$ with respect to the reference direction $\Delta$. In FTIR experiments of uniaxially deformed samples, u represents the transition moment vector, and $\Delta$ is the stretch direction. The angle between $\mathbf{u}$ and $\Delta$ is shown as $\chi$. (b) Angular position $\vartheta$ of the chain-embedded molecular axis $\xi$, with respect to $\Delta$. u makes an angle $\alpha$ with the molecular axis $\xi$. Here the direction of $\xi$ is taken along that of the vector connecting backbone atoms $i$ and $i+2$. (c) Local coordinate system of axes $\xi, \eta$, and $\zeta$ defining the position of $u$ with respect to the chain backbone. The $\eta$-axis is chosen in the plane of the molecular axis $\xi$ and the laboratory fixed axis, $\Delta$. The $\zeta$-axis completes the orthogonal system. The arimuthal angle $\alpha$ and the polar angle $\varphi$, define the orientation of $u$ with respect to the system.

copolymeric chains composed of monomeric units which possess different geometrical and conformational character will be considered, and segmental orientation simultaneously occurring in either component of the chain will be analyzed. The plan of the paper is as follows: In part II, the measure of segmental orientation is defined. The conventional treatment of data, such as those from infrared or NMR experiments, is briefly outlined. In part III, calculation of segmental orientation by the matrix generation technique based on rotational isomeric state (RIS) formalism and by Monte Carlo simulations is described. In part IV, results of calculations for $\mathrm{AB}$ type alternating copolymers are reported, showing the importance of local conformational and geometric features on segmental orientation. Concluding remarks are presented in part V.

\section{Description of Segmental Orientation and} Interpretation in Terms of FTIR Experiments

a. Definition of Segmental Orientation. A vector u rigidly affixed to a bond of a polymer chain is shown in Figure 1a. The dashed line identified as $\Delta$ and making an angle $\chi$ with $u$ represents a laboratory-fixed axis. In uniaxial deformation, $\Delta$ coincides with the direction of the applied load which may be tensile or compressive. The orientation of $\mathbf{u}$ with respect to $\Delta$ is expressed in terms of the orientation function $S(\chi)$, which is given by the second Legendre polynomial $P_{2}(\cos \chi)$ as

$$
S(\chi)=\left\langle P_{2}(\cos \chi)\right\rangle=(1 / 2)\left(3\left\langle\cos ^{2} \chi\right\rangle-1\right)
$$

The angular brackets refer to the ensemble averaging over all configurations of the chain. In FTIR experiments, the measured dichroism directly gives $S(\chi)$ as described in the following section. Similarly in ${ }^{2} \mathrm{H}-\mathrm{NMR}$ experiments, measured splitting leads ${ }^{3}$ to $S(\chi)$.

In addition to the orientation of the label $\mathbf{u}$, the orientation of another axis, called the "molecular axis" embedded into a portion of the chain may be of interest. An example to such a molecular axis is shown in Figure $1 b$, by the dashed line $\xi$ which forms an angle $\alpha$ and $u$ and an angle $\vartheta$ with the laboratory fixed axis $\Delta$. In general, the chain segment characterizing the molecular axis $\xi$ is chosen to include portions of the chain ranging from a single bond to a few monomeric units, depending on the vectorial quantity probed by the specific experimental technique. In Figure 1b, the chain segment consists of two bonds containing the label $u$. The orientation of the chain segment or equivalently of the molecular axis is expressed by the orientation function $S(\vartheta)$ defined according to eq 1 with $\vartheta$ replacing $\chi$.

b. Segmental Orientation in Uniaxially Deformed Networks. In statistical calculations according to the RIS scheme and the phantom network theory of elasticity, the orientation $S(\chi)$ of the vector u relative to the direction of stretch is obtained from the expression

$$
S(\chi)=D_{0}(1-2 / f)\left(\lambda^{2}-\lambda^{-1}\right)
$$

where $\lambda$ is the extension ratio defined as the ratio of the final macroscopic length of the network under uniaxial extension to its length in the reference state, $f$ is the junction functionality, and $D_{0}$ is the configurational factor defined by

$$
D_{0}=\left(3\left\langle r^{2} \cos ^{2} \Phi\right\rangle_{0} /\left\langle r^{2}\right\rangle_{0}-1\right) / 10
$$

Here, $\Phi$ is the angle between $u$ and the chain end-to-end vector $\mathbf{r}$. The subscript zero indicates that the averaging is performed for chains in the unconstrained state. The first molecular approach to the problem of segmental orientation in real chains, not necessarily conforming with the freely jointed chain model, is the pioneering work of Nagai, ${ }^{19}$ which was further developed by Flory. ${ }^{5}$ The rotational isomeric state (RIS) approximation of chain statistics is adopted in these formulations, leading to an expression for $S$ in the form of a series expansion containing various order moments of the chain end-to-end vector and related trigonometric variables. For sufficiently long chains and small deformations, the first term of the series represented by eq 2 is adequate. For finite chains subject to large deformations, the reader is referred to a recent extension ${ }^{20}$ of the formulation of Nagai and Flory in which explicit expression including higher order terms is presented.

For a vector lying along the backbone of a sufficiently long chain, the front factor $D_{0}$ equates to $1 / 5 N$, where $N$ is the number of Kuhn segments in the chain. For network chains of $n$ bonds, within the validity of the RIS scheme, eq 2 represents the exact first term, linear in $n^{-1}$, replacing the front factor $1 / 5 \mathrm{~N}$ of the Kuhn approximation. The moments $\left\langle r^{2}\right\rangle_{0}$ and $\left\langle r^{2} \cos ^{2} \Phi\right\rangle_{0}$ in eq 3 are readily calculated with the RIS matrix generation technique whereas the higher order moments appearing in the serial expansion ${ }^{20}$ for $S(\chi)$ are estimated by Monte Carlo (MC) chain generation.

c. Description of Orientation in FTIR Experiments. Fourier transform infrared experiments measure the absorption bands associated with transition moments having a definite orientation with respect to the chain backbone. The vector $u$ of Figure 1 which is rigidly affixed 
to a chain bond is assumed to be the transition moment vector. The relative orientation of $\mathbf{u}$ with respect to the chain backbone, which is along the axis $\xi$, is defined by the azimuthal angle $\alpha$ and the polar angle $\varphi$, with respect to the coordinate system shown in Figure 1c. In this figure, the $\eta$ and $\zeta$-axes are chosen within a plane whose normal is the molecular axis, $\xi$.

For incident radiation polarized along $\Delta$, the measured absorbance due to the particular orientation of $u$ may be resolved into a component parallel, $a_{\|}$, and another perpendicular, $a_{\perp}$, to $\Delta$ as

$$
a_{\|}=|\mathbf{u}|^{2} \cos ^{2} \chi
$$

and

$$
a_{\perp}=(1 / 2)|\mathbf{u}|^{2} \sin ^{2} \chi
$$

The factor of $1 / 2$ in eq 5 results from averaging of all rotations of $\mathbf{u}$ about the stretch direction $\Delta$, which constitutes an axis of cylindrical symmetry. The dichroic ratio $R$ measured in infrared studies is defined as

$$
R=\int_{0}^{\pi} a_{\sharp} h(\chi) \sin \chi \mathrm{d} \chi / \int_{0}^{\pi} a_{\perp} h(\chi) \sin \chi \mathrm{d} \chi
$$

where $h(\chi)$ represents the distribution of the angle $\chi$. Substituting eqs 4 and 5 in eq 6 and using eq 1 leads to

$$
S(\chi)=\frac{R-1}{R+2}
$$

On the other hand, the orientation of the local chain axis $\xi$ with respect to the direction of stretch is given by

$$
S(\vartheta)=\frac{2}{3 \cos ^{2} \alpha-1} \frac{R-1}{R+2}
$$

This equation is obtained by expressing $a_{\|}$and $a_{\perp}$ in terms of $\alpha, \vartheta$, and $\varphi$, averaging over $\varphi$ by assuming cylindrical symmetry about the axis $\xi$ and finally integrating over all $\vartheta$ directions using a distribution function $f(\vartheta)$. Equation 8 may be rearranged using eq 7 as

$$
S(\chi)=S(\vartheta) S(\alpha)
$$

where $S(\alpha)=(1 / 2)\left(3 \cos ^{2} \alpha-1\right)$. This form was first given by Fraser ${ }^{21}$ and applied to orientation in deformed polymers by Read and Stein. ${ }^{22}$ In the latter work, the angle $\vartheta$ has been referred to as the angle between the stretch direction and the "segments" of the chains. That the directions of these segments should be the axes of cylindrical symmetry for the associated transition moments measured in the experiments was not stated explicitly.

Equation 9 shows that the dichroic ratio measurements depend (i) on the particular orientation of the transition moment vectors relative to the local molecular axis, if such an axis of cylindrical symmetry can be defined on a local scale, and (ii) on the mean-square projection of that axis along the stretch direction. However, the anisotropy of chain structure and of rotational isomeric states might invalidate the adoption of an axis of cylindrical symmetry on a local scale. Then, the integration over the polar angle $\varphi$ should be carried out by adopting a joint distribution function $f(\vartheta, \varphi)$ for the coupled change of the two degrees of freedom. Thus, in a given chain, the different segments along the backbone, possessing different geometry and conformational characteristics, may lead to different dichroic ratio values, even if the corresponding transition

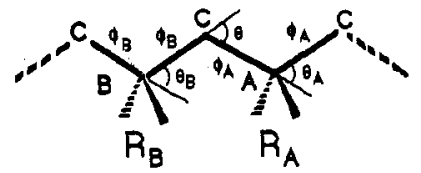

Figure 2. Schematic representation of a portion of an alternating copolymer with two types of monomeric units -[AC]- and -[BC]-, and substituents $R_{\mathrm{A}}$ and $R_{\mathrm{B}}$ attached on atoms $\mathrm{A}$ and $B$, respectively. All bonds are of equal length. The supplemental bond angles are denoted as $\theta, \theta_{A}$, and $\theta_{B}$ depending on the type, $\mathrm{C}, \mathrm{A}$, or $\mathrm{B}$, of the backbone atom. Bond torsional rotations are indicated by $\phi_{A}$ for bonds $C A$ and $A C$ and by $\phi_{B}$ for bonds $C B$ and $\mathrm{BC}$.

moments are at the same fixed angular position with respect to the backbone.

From the preceding arguments the following important conclusion may be drawn: The local structural and conformational characteristics of the chain play a major role in prescribing the molecular orientation, and a quantitative estimation of the latter requires consideration of the detailed structure of chain segments. This will be the subject of the succeeding section.

\section{Calculation of Segmental Orientation in Copolymers}

The configurational averages $\left\langle r^{2} \cos ^{2} \Phi\right\rangle_{0}$ and $\left\langle r^{2}\right\rangle_{0}$ appearing in the expression (eq 3 ) for the configurational factor $D_{0}$ have been calculated by both matrix generation technique based on RIS formalism and MC simulations.

(a) Model Copolymer. The copolymer is assumed to be composed of two types of monomeric units $A$ and $B$, comprising each two backbone bonds, with different substituents $R_{\mathrm{A}}$ and $R_{\mathrm{B}}$ attached on alternating backbone atoms. Backbone bonds flanking atom $A$ are indicated as $\mathrm{CA}$ and $\mathrm{AC}$, those about $\mathrm{B}$ are $\mathrm{CB}$ and $\mathrm{BC}$, all of them being of equal length $l_{0}$. The supplemental bond angles are denoted as $\theta, \theta_{\mathrm{A}}$, and $\theta_{\mathrm{B}}$ depending on the type, $\mathrm{C}, \mathrm{A}$, or $B$, respectively, of the backbone atom, as shown in Figure 2. Bond torsional rotations are indicated by the variable $\phi_{\mathrm{A}}$ for bonds CA and AC, and by $\phi_{\mathrm{B}}$ for bonds CB and BC. Three discrete rotational isomeric states, centered about the conventional trans $(\mathrm{t})$, gauch $e^{+}\left(\mathrm{g}^{+}\right)$, and gauche $\left.\mathrm{e}^{-} \mathrm{g}\right)$ states are accessible to backbone bonds. Such a geometric representation conforms with polystyrene-poly(methyl methacrylate) copolymers, for example. Calculations are carried out for independent as well as pairwise interdependent rotational states for neighboring bonds along the chain.

The vector $\mathbf{u}$, whose orientation is of interest, is chosen to be either along the backbone bonds $\left(\mathbf{u}_{\|}\right)$, or perpendicular to the line connecting two successive $\mathrm{C}$ atoms lying in the plane defined by the two bonds between those $\mathrm{C}$ atoms $\left(\mathbf{u}_{\perp}\right)$. The unit vectors $\mathbf{u}_{\|}$along bonds $C A$ and $A C$ are indicated as $u_{\|}{ }^{A}$ and those along $C B$ and $B C$ as $u_{\|}$. Likewise, superscripts $A$ and $B$ are appended to $u_{\perp}$ in order to indicate the monomeric unit to which they belong. Thus, two sets of vectors (i) $\mathbf{u}_{\|}^{A}$ and $\mathbf{u}_{\|}^{B}$ and (ii) $\mathbf{u}_{\perp}{ }^{A}$ and $\mathbf{u}_{\perp}{ }^{B}$ are analyzed. Results are obtained by both matrix generation method based on RIS formalism and Monte Carlo chain generation. The two sets (i) and (ii) exhibit substantially distinct $D_{0}$ values, the former being positively oriented with respect to $r$ and the latter exhibiting negative orientation (negative $D_{0}$ or $S$ ), as might be intuitively expected from the identity $\Sigma_{i}\left\langle\cos ^{2} \Phi_{i}\right\rangle=1$ where $\Phi_{i}$ is the angle between $u$ and the $i$ th axis of a Cartesian laboratory fixed frame.

In computations, fluctuations of amplitude $\pm 40^{\circ}$ are allowed in the location of isomeric minima as well as in 
the bond angles, and the effects of geometry and energy parameters on the orientation of vectors $\mathbf{u}_{\|}$and $\mathbf{u}_{\perp}$ rigidly embedded in either of the monomeric units $A$ or $B$ are studied.

(b) Mathematical Methods for Evaluating $D_{0}$. Inasmuch as the segmental orientation may be written in terms of eq 2 as a first approximation to real chain behavior, the calculation of $S$ for a given extension ratio reduces to that of the front factor $D_{0}$, as the moments $\left\langle r^{2} \cos ^{2} \Phi\right\rangle_{0}$ and $\left\langle r^{2}\right\rangle_{0}$ in eq 3. Precise evaluation of these moments is critically important, because only slight departures from isotropic behavior $\left\langle r^{2} \cos ^{2} \Phi\right\rangle_{0} /\left\langle r^{2}\right\rangle_{0}=\left\langle\cos ^{2} \Phi\right\rangle=1 / 3$ are responsible for the observed behavior. Accordingly, the matrix generation method which yields exact results for $\left\langle r^{2} \cos ^{2} \Phi\right\rangle_{0}$ and $\left\langle r^{2}\right\rangle_{0}$ are adopted. On the other hand, part of the calculations are repeated using Monte Carlo chain generation method in order to assess the applicability of this approximate method which would become indispensable in evaluating higher order moments. A brief recapitulation of both methods is presented below. The reader is referred to original references for further details of the mathematical methods.

Matrix Generation Technique. The moment $\left\langle r^{2} \cos ^{2}\right.$ $\Phi\rangle_{0}$ associated with the orientation of a reference vector affixed to the $i$ th unit along the chain is calculated ${ }^{5,23}$ by the RIS scheme using

$$
\left\langle r^{2} \cos ^{2} \Phi\right\rangle_{0}=\mathbf{J} *\left(\prod_{k=1}^{i-1} \mathfrak{F}_{k}{ }^{\prime}\right) \mathcal{F}_{i}\left(\prod_{k=i+1}^{n} \mathcal{F}_{k}{ }^{\prime}\right) \mathbf{J} Z^{-1}
$$

where $Z$ is the configurational partition function, $\mathbf{J}^{*} \equiv$ $\operatorname{row}(1,0,0, \ldots), \mathbf{J} \equiv \operatorname{col}(1,1,1, \ldots), \mathcal{F}_{i}$ is defined for the sequence of four consecutive bonds as

$$
\mathfrak{F}_{\mathrm{i}}=\mathscr{F}_{\mathrm{i}}^{\mathrm{CA}} \mathcal{F}_{\mathrm{i}}^{\mathrm{AC}} \boldsymbol{F}_{\mathrm{i}}^{\mathrm{CB}} \mathcal{F}_{\mathrm{i}}^{\mathrm{BC}}
$$

Here, $\mathbf{F}_{i}^{\mathrm{X}}$ where $\mathrm{X}=\mathrm{CA}, \mathrm{AC}, \mathrm{CB}$, and $\mathrm{BC}$ is evaluated from

$$
\mathcal{F}_{i}^{\mathrm{X}}=\left(\mathbf{U}_{i}^{\mathrm{X}} \otimes \mathbf{E}_{26}\right)\left\|\mathbf{F}_{i}^{\mathrm{X}}\right\|
$$

where $\mathbf{E}_{26}$ is identity matrix of order $26, \otimes$ is the direct product, $\mathbf{U}_{i}$ is the statistical weight matrix which is taken to be of the form

$$
\mathbf{U}_{i}^{\mathrm{X}}=\left[\begin{array}{lll}
1 & \sigma_{\mathrm{X}} & \sigma_{\mathrm{X}} \\
1 & \sigma_{\mathrm{X}} & \sigma_{\mathrm{X}} \omega_{\mathrm{X}} \\
1 & \sigma_{\mathrm{X}} \omega_{\mathrm{X}} & \sigma_{\mathrm{X}}
\end{array}\right]
$$

and $\left\|\mathbf{F}_{i} \mathbf{X}\right\|$ is the matrix composed of the diagonal arrays of the generating matrix $\mathbf{F}_{i}$ of states trans $(t)$, gauche ${ }^{+}$ $\left(\mathrm{g}^{+}\right)$, and gauche ${ }^{-}(\mathrm{g})$ for the particular bond X. $\mathrm{F}_{i}$ is given by eq 9 of ref 23 , in which $\mathbf{m}_{i}$ is replaced by $\mathbf{u}_{\|}{ }^{A}, \mathbf{u}_{\|}{ }^{B}$, $\mathbf{u}_{\perp}{ }^{A}$, or $\mathbf{u}_{\perp}{ }^{B}$, depending on the investigated vector. $\sigma_{\mathbf{X}}$ refers to the first-order interaction parameter associated with the $g^{ \pm}$state of bond X; $\omega \mathrm{X}$ is the second-order interaction parameter corresponding to $g^{ \pm} g^{\mp}$. We note that both $\omega_{\mathrm{CB}}$ and $\omega_{\mathrm{CA}}$ give rise to the interaction of two $\mathrm{C}$ atoms separated by four bonds and therefore will be taken to be equal to each other. $\omega_{\mathrm{BC}}$ and $\omega_{\mathrm{AC}}$ describe the interactions between successive $A$ and $B$ atoms, respectively. $\omega_{X}$ is set equal to 1 in the case of independent bonds. In the extreme case of infinitely large repulsion, $\omega \mathrm{X}=0$.
Similarly, the mean-squared end-to-end distance $\left\langle r^{2}\right\rangle_{0}$ is calculated according to the matrix generation method using 5

$$
\left\langle r^{2}\right\rangle_{0}=Z^{-1} \mathbf{J}^{*}\left(\prod_{i=1}^{n} \Theta_{i}\right) \mathbf{J}
$$

where

$$
\mathcal{S}_{i}=\mathcal{g}_{i}^{\mathrm{CA}} \mathcal{g}_{i}^{\mathrm{AC}} g_{i}^{\mathrm{CB}} g_{i}^{\mathrm{BC}}
$$

with

$$
g_{i}^{\mathrm{X}}=\left(\mathbf{U}_{i} \otimes \mathbf{E}_{5}\right) \| \mathbf{G}_{i} \mathbf{X}_{\|}
$$

and $\left\|G_{i} \mathbf{X}\right\|$ is the diagonal array of the generator matrices $\mathrm{G}_{i}$ of states trans $(\mathrm{t})$, gauche $e^{+}\left(\mathrm{g}^{+}\right)$, and gauche $e^{-}(\mathrm{g})$ for the particular bond $\mathrm{X}$. The generator matrix $\mathrm{G}_{i} \mathrm{X}$ has the familiar form given in ref 5 .

Monte Carlo Generation. In MC chain generation bond dihedral angles are assigned on the basis of the probability distributions of independent rotational states for each bond. From symmetry considerations, the equilibrium probabilities of bond rotational states $t, \mathrm{~g}^{+}$, and $g-$ obey the relationships

$$
\begin{array}{cl}
p_{\mathrm{CA}}(\mathrm{t})=p_{\mathrm{AC}}(\mathrm{t}) ; & p_{\mathrm{CB}}(\mathrm{t})=p_{\mathrm{BC}}(\mathrm{t}) \\
p_{\mathrm{CA}}\left(\mathrm{g}^{+}\right)=p_{\mathrm{AC}}\left(\mathrm{g}^{-}\right) ; & p_{\mathrm{CB}}\left(\mathrm{g}^{+}\right)=p_{\mathrm{BC}}\left(\mathrm{g}^{-}\right) \\
p_{\mathrm{CA}}\left(\mathrm{g}^{-}\right)=p_{\mathrm{AC}}\left(\mathrm{g}^{+}\right) ; & p_{\mathrm{CB}}\left(\mathrm{g}^{-}\right)=p_{\mathrm{BC}}\left(\mathrm{g}^{+}\right)
\end{array}
$$

For independent bonds, the total number of equilibrium probabilities to specify the configurational distribution of rotameric states in the copolymer reduces from 12 to 4 , using the two linearly independent identities $p_{\mathrm{CB}}(\mathrm{t})+p_{\mathrm{CB}}-$ $\left(\mathrm{g}^{+}\right)+p_{\mathrm{CB}}\left(\mathrm{g}^{-}\right)=1$ and $p_{\mathrm{CA}}(\mathrm{t})+p_{\mathrm{CA}}\left(\mathrm{g}^{+}\right)+p_{\mathrm{CA}}(\mathrm{g})=1$, in addition to eq 17. The set of variables $p_{\mathrm{CB}}(\mathrm{t}), p_{\mathrm{CA}}(\mathrm{t})$, $p_{\mathrm{CA}}(\mathrm{g})$, and $p_{\mathrm{CB}}(\mathrm{g})$ will be varied in the calculations.

The following steps are performed for each generated chain: (i) the chain vector $\mathbf{r}$ between terminal atoms is calculated. (ii) The angles $\Phi_{i}{ }^{A}$ and $\Phi_{i}{ }^{B}$ between the vector $\mathbf{r}$ and the transition moment vectors $\mathbf{u}_{i}{ }^{A}$ and $\mathbf{u}_{i}{ }^{B}$, appended to the $i$ th unit of type $A$ and $B$, respectively, is found from $\Phi_{i}^{x}=\cos ^{-1}\left[\left(u_{i}^{x} \cdot \mathbf{r}\right) /\left(u_{i}^{x} r\right)\right] . u_{i}^{x}$ and $r$ are the magnitudes of $\mathbf{u}_{i}{ }^{x}$ and $\mathbf{r}$, respectively. The dot denotes the scalar product and $x$ stands for either $A$ or $B$. (iii) the arithmetic average $\cos ^{2} \Phi_{X}$ over all units of a particular type $\mathrm{x}$ is calculated for the generated chain, the terminal three units at both ends being excluded. (iv) the values $r^{2}=(\mathbf{r} \cdot \mathbf{r})$ and $r^{2} \cos ^{2} \Phi_{\mathrm{X}}$ are recorded. (v) Steps i-iv are repeated for $10^{5}$ Monte Carlo chains and the averages $\left\langle r^{2}\right\rangle_{0}$ and $\left\langle r^{2} \overline{\cos ^{2} \Phi_{X}}\right\rangle_{0}$ over all chains are inserted into eq 3 , to evaluate $D_{0 X}$ for $\mathrm{X}=\mathrm{CA}$ and $\mathrm{CB}$.

\section{Results of Calculations}

Influence of Bond Angles. For an assessment of the influence of bond angles on segmental orientation, $\theta_{\mathrm{B}}$ has been varied while $\theta_{\mathrm{A}}$ and $\theta$ were held fixed at the tetrahedral value. Calculations were performed for the two cases of (i) rotationally independent and (ii) pairwise interdependent bonds, assuming identical length and energy parameters for units $A$ and $B$. In the case of interdependent 


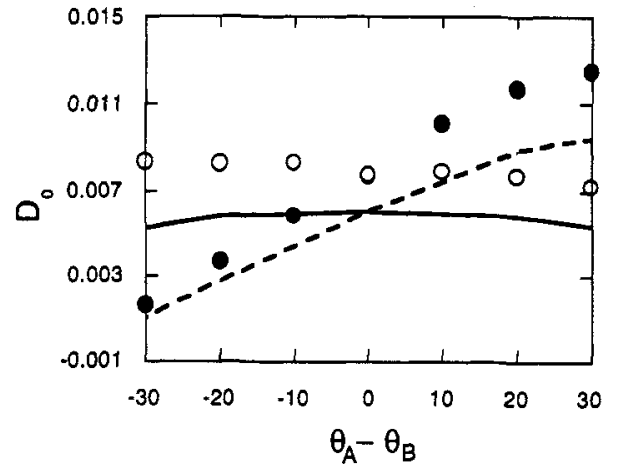

Figure 3. Configurational factors $D_{0 \mathrm{~A}}$ and $D_{0 \mathrm{~B}}$ obtained for unit vectors $\mathbf{u}_{\|}^{A}$ and $\mathbf{u}_{l}^{B}$ along backbone bonds, as a function of $\theta_{B}$. The solid and dashed curves are calculated for $u_{\|}^{A}$ and $u_{B}^{B}$, respectively, using a matrix multiplication method of the RIS scheme for chains with independent bonds. $p_{\mathrm{CB}}(\mathrm{t})=p_{\mathrm{CA}}(\mathrm{t})=$ 0.48 and $p_{\mathrm{CA}}(\mathrm{g})=p_{\mathrm{CB}}(\mathrm{g})=0.26, \theta_{\mathrm{A}}=109.5^{\circ}$, and $\phi(\mathrm{t})=0^{\circ}$ and $\phi\left(g^{ \pm}\right)= \pm 120^{\circ}$ for all bonds. The empty and filled circles represent the average over $10^{5}$ Monte Carlo chains. Bond angles are the only parameters differing in the two monomeric units of the copolymer, as indicated in the abscissa.

rotational states, both favorable and unfavorable secondorder interactions have been considered.

The results can be summarized as follows: First, the orientation of $\mathbf{u}_{\|}$in the two types of monomers have been analyzed for chains with rotationally independent bonds. In the particular case of $\theta_{\mathrm{A}}=\theta_{\mathrm{B}}$ the transition moments with a given orientation with respect to the local chainembedded frame, appended to atoms $A$ and $B$, naturally exhibit same segmental orientation, manifested by identical values $D_{0 \mathrm{~A}}=D_{0 \mathrm{~B}}$. Figure 3 displays the configurational factors $D_{0 \mathrm{~A}}$ and $D_{0 \mathrm{~B}}$ obtained from both $\mathrm{MC}$ simulations and matrix generation for independent bond rotations, using $\mathbf{u}_{\|}{ }^{A}$ and $\mathbf{u}_{\|}^{B}$. The abscissa in the figure represents the difference between the two bond angles $\theta_{\mathrm{A}}$ and $\theta_{\mathrm{B}}$. Simulation results are presented by the empty and filled circles for $D_{0 \mathrm{~A}}$ and $D_{0 \mathrm{~B}}$, respectively, and the labeled curves which fall generally below the $\mathrm{MC}$ results are calculated by the RIS matrix multiplication scheme. The results are obtained for $p_{\mathrm{CB}}(\mathrm{t})=p_{\mathrm{CA}}(\mathrm{t})=0.48$ and $p_{\mathrm{CA}}\left(\mathrm{g}^{-}\right)=p_{\mathrm{CB}}(\mathrm{g})=0.26$, with the torsional states $\phi(\mathrm{t})=$ $0^{\circ}$ and $\phi\left(\mathbf{g}^{ \pm}\right)= \pm 120^{\circ}$ for all bonds. We note that for polymers composed of monosubstituted monomers, chain asymmetry implies distinct probabilities for the $\mathrm{g}^{+}$and $\mathrm{g}$ states of a given bond, with a given chirality. By adopting equal probabilities for those two states, we implicitly consider the average statistical behavior of atactic units, with substituents attached in either $l$ or $d$ positions.

We observe in Figure 3 that the changes in $\theta_{B}$ values do not perturb the orientation of $\mathbf{u}_{\|}{ }^{A}$ as evidenced from the approximately horizontal curve obtained for $D_{0 \mathrm{~A}}$ while $D_{0 \mathrm{~B}}$ exhibits some smooth increase with decreasing $\theta_{\mathrm{B}}$. The insensitivity of $D_{0 \mathrm{~A}}$ to $\theta_{\mathrm{B}}$ is expected since the energy and length parameters of unit $A$ are kept fixed in obtaining Figure 3. It should be pointed out, that the adoption of $\theta_{\mathrm{B}}=50^{\circ}$, which is a reasonable equilibrium value for units with large substituents such as $\mathrm{Si}-\mathrm{O}-\mathrm{Si}$ bond angles in poly(dimethylsiloxane), leads to a $50 \%$ increase in the configurational factor, $D_{0}$, from $\sim 0.0052$ to 0.010 , which is directly reflected upon the orientation function $S$. Thus, in spite of the identical conformational probability distribution of the isomeric states of the two monomeric units, and the inherent constraints imposed by chain connectivity, the bond vectors of the two monomeric units exhibit quite distinct orientations, this arising only from a slight perturbation in the bond angles of one of the units.

Calculations repeated for pairwise interdependent bonds but not shown in Figure 3 demonstrated the following: If

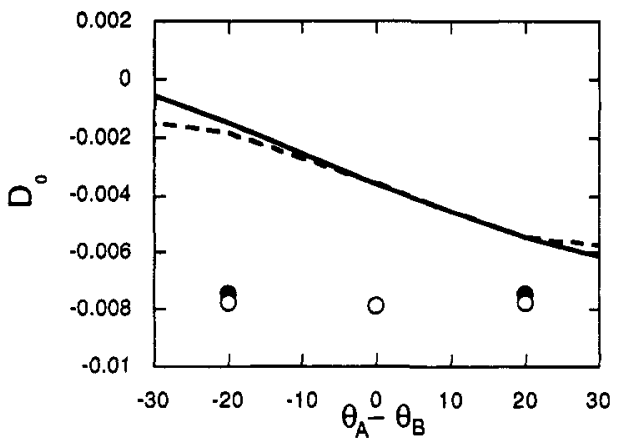

Figure 4. Configurational factors $D_{0 \mathrm{~A}}$ and $D_{0 \mathrm{~B}}$ obtained for $\mathbf{u}_{\perp} \mathrm{A}$ and $u_{\perp}{ }^{B}$, using the same model and parameters as in Figure 3. $\mathbf{u}_{\perp} \mathbf{x}$ is the unit vector perpendicular to the plane defined by bonds CX and XC, with $\mathrm{X}=\mathrm{A}$ or $\mathrm{B}$. See legend to Figure 3 .

both $A-A$ and $B-B$ pairs are subject to unfavorable secondorder interactions of the same strength $\left(\omega_{\mathrm{BC}}=\omega_{\mathrm{AC}}<1\right)$, while $\mathrm{C}-\mathrm{C}$ pairs do not experience any second-order interaction $\left(\omega_{\mathrm{CA}}=\omega_{\mathrm{CB}}=1\right)$, the deviation between $D_{0 \mathrm{~A}}$ and $D_{0 \mathrm{~B}}$ is enhanced. In the opposite case of attractive second-order interactions $\left(\omega_{\mathrm{BC}}=\omega_{\mathrm{AC}}>1\right)$, the difference between $D_{0 \mathrm{~A}}$ and $D_{0 \mathrm{~B}}$ diminishes.

The dependence of $\mathbf{u}_{\perp}{ }^{A}$ and $\mathbf{u}_{\perp}{ }^{B}$ on $\theta_{A}-\theta_{B}$ is shown in Figure 4 for a chain with independent rotational states. The curves show results of exact calculations. The orientational behavior of $\mathbf{u}_{\perp}{ }^{A}$ and $\mathbf{u}_{\perp}{ }^{B}$ is markedly different than that for $\mathbf{u}_{\|}$. In contrast to $\mathbf{u}_{\|}$, the orientations of the perpendicular vectors $\mathbf{u}_{\perp}{ }^{A}$ and $\mathbf{u}_{\perp}{ }^{B}$ do not differ much from each other as $\theta_{B}$ is varied. Yet, both $u_{\perp}{ }^{A}$ and $\mathbf{u}_{\perp}{ }^{B}$ exhibit a strong dependence on $\theta_{A}-\theta_{B}$ as observed from the figure. The empty and filled circles shown in the figure represent results of $\mathrm{MC}$ simulations. The agreement between analytical curves and MC results is poor, which demonstrates the inadequacy of the $\mathrm{MC}$ method for calculating the orientation of $\mathbf{u}_{\perp}$.

The effect of bond interdependence on the orientation of $\mathbf{u}_{\perp}{ }^{A}$ and $\mathbf{u}_{\perp}{ }^{B}$ is shown in Figure 5. In part $\mathbf{a}$ of the figure, backbone atoms $A$ are strongly attracted $\left(\omega_{B C}=0\right)$, whereas a strong repulsion is imposed between atoms $B$ of the backbone $\left(\omega_{A C}=10\right)$. No second-order interaction occurs between $\mathrm{C}-\mathrm{C}$ pairs. The opposite situation $\left(\omega_{\mathrm{BC}}=\right.$ $10, \omega_{\mathrm{AC}}=0$ ) applies to Figure $5 \mathrm{~b}$ ). Incorporation of interactions between backbone atoms results in strong perturbations in the orientation of $\mathbf{u}_{\perp}{ }^{B}$, especially for positive values of $\theta_{\mathrm{A}}-\theta_{\mathrm{B}}$. It is to be noted that the configurational factor for $\mathbf{u}_{\perp}{ }^{B}$ may take large positive values (Figure 5a) or negative values (Figure 5b), depending on the type of interaction between backbone bonds.

Influence of Rotational States. Here we turn our attention to the role of the location of the rotational minima in prescribing segmental orientation. For this purpose, the minimum energy dihedral angle representative of the gauch $e^{ \pm}$state is assumed to deviate within $\pm 40^{\circ}$ from the perfect staggered position given by $\pm 120^{\circ}$. All bond angles are constrained to tetrahedral values. The probability distributions of rotameric states of the two types of monomeric units are the same, although $\mathrm{g}^{ \pm}$states are now assumed in both units to be more favorable. This assumption is adopted for a clear visualization of the effect of angular distortions in the $\mathrm{g}^{ \pm}$states.

Figure 6 displays the configurational factors obtained with $p_{\mathrm{CB}}(\mathrm{t})=p_{\mathrm{CA}}(\mathrm{t})=0.2$ and $p_{\mathrm{CA}}(\mathrm{g})=p_{\mathrm{CB}}(\mathrm{g})=0.4$, for the vectors $u_{\|}$for a chain with independent bonds. The curves are obtained from matrix generation calculations and circles are from $\mathrm{MC}$ simulations. The rotameric states of bonds CA and AC are held fixed at the $\phi_{\mathrm{A}}($ trans $)=0^{\circ}$, $\phi_{\mathrm{A}}\left(\right.$ gauche $\left.^{ \pm}\right)= \pm 120^{\circ}$, while the gauche states of bonds 

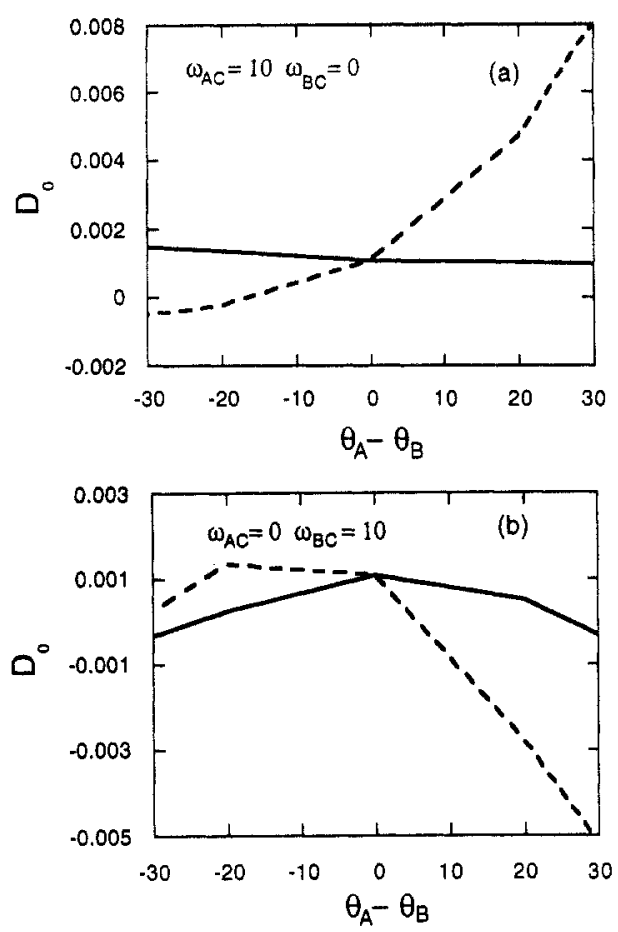

Figure 5. Configurational factors $D_{0 \mathrm{~A}}$ and $D_{0 \mathrm{~B}}$ obtained for $\mathbf{u}_{\perp} \mathrm{A}$ and $u_{\perp}{ }^{B}$ by RIS scheme, using the same energy and length parameters as in Figure 3, except for the second-order interaction parameters: (a) $\omega_{A C}=10, \omega_{B C}=0 ;$ (b) $\omega_{A C}=0, \omega_{B C}=10$. See legend to Figure 3.

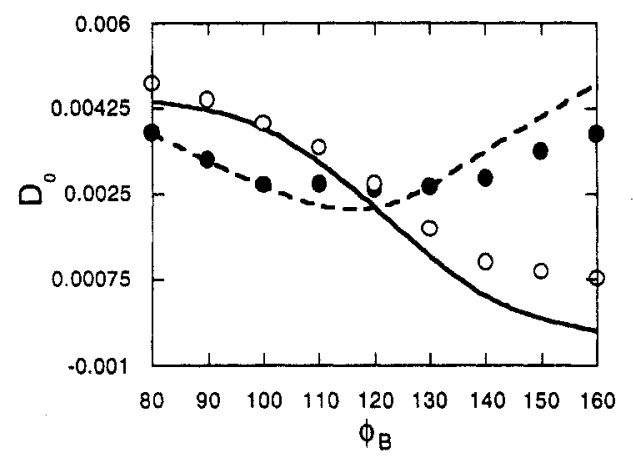

Figure 6. Influence of the location of the rotational isomeric states on the configurational factors $D_{0 \mathrm{~A}}$ and $D_{0 \mathrm{~B}}$ associated with $\mathrm{u}_{1}{ }^{\wedge}$ and $\mathrm{u}_{\mathrm{B}}^{\mathrm{B}}$ for $p_{\mathrm{CB}}(\mathrm{t})=p_{\mathrm{CA}}(\mathrm{t})=0.2$ and $p_{\mathrm{CA}}(\mathrm{g})=p_{\mathrm{CB}}(\mathrm{g})=0.4$ All second-order interaction parameters are taken as unity. Solid and dashed curves represent results of exact calculations for units $A$ and $B$, respectively. Empty and filled circles are obtained by MC simulations by averaging over $10^{5} \mathrm{MC}$ chains. The rotameric states of the bonds in unit $A$ are held fixed at the values $\phi_{A^{-}}$ (trans) $=0^{\circ}, \phi_{\mathrm{A}}$ (gauche $\left.e^{ \pm}\right)= \pm 120^{\circ}$, while the gauche states of bonds in unit $B$ are varied, as shown in the abscissa.

$\mathrm{BC}$ and $\mathrm{CB}$ are varied, as shown in the abscissa. $D_{0 \mathrm{~B}}$ exhibits a relatively weak perturbation, due to the changes in $\phi_{\mathrm{A}}$, but the changes in $D_{0 \mathrm{~A}}$ are more pronounced. $\mathrm{A}$ shift of $\pm 20^{\circ}$ in the location of the $\mathrm{g}^{ \pm}$state of $\mathrm{BC}$ and $\mathrm{CB}$ bonds, for instance, leads to about 1 order of magnitude difference between the segmental orientations of the two units. In fact, $D_{0 \mathrm{~B}}=0.0035$ and $D_{0 \mathrm{~A}} \approx 0.0005$ when $\phi_{\mathrm{A}}\left(\mathrm{g}^{ \pm}\right)$ $= \pm 140^{\circ}$, whereas at $\phi_{\mathrm{A}}\left(\mathrm{g}^{ \pm}\right)=120^{\circ}$ both $D_{0 \mathrm{~B}}$ and $D_{0 \mathrm{~A}}$ equate to 0.0022 . An interesting observation is the highly nonlinear response of the configurational factors to the changes in the location of isomeric minima.

In Figure 7, the dependence of the configurational factors for $\mathbf{u}_{\perp}{ }^{A}$ and $\mathbf{u}_{\perp}{ }^{B}$ on the changes in $\phi_{B}$ is shown. The curve represents results of matrix generation calculations and the circles are from MC simulations. Matrix generation calculations lead to identical results for the orientation of $\mathbf{u}_{\perp}{ }^{A}$ and $\mathbf{u}_{\perp}{ }^{B}$ as identified by the single curve in the figure.

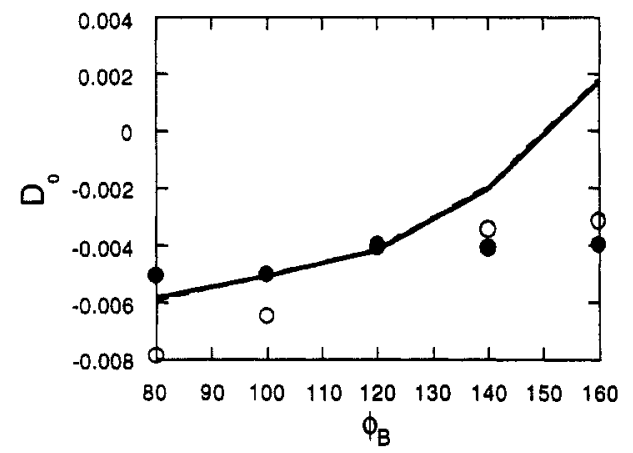

Figure 7. Influence of rotational isomeric states on the configurational factors $D_{0 \mathrm{~A}}$ and $D_{0 \mathrm{~B}}$ obtained for $\mathbf{u}_{\perp}{ }^{A}$ and $\mathbf{u}_{\perp}{ }^{B}$, using the same parameters as in Figure 6 . Exact results for units $A$ and $B$ are shown by the same curve.
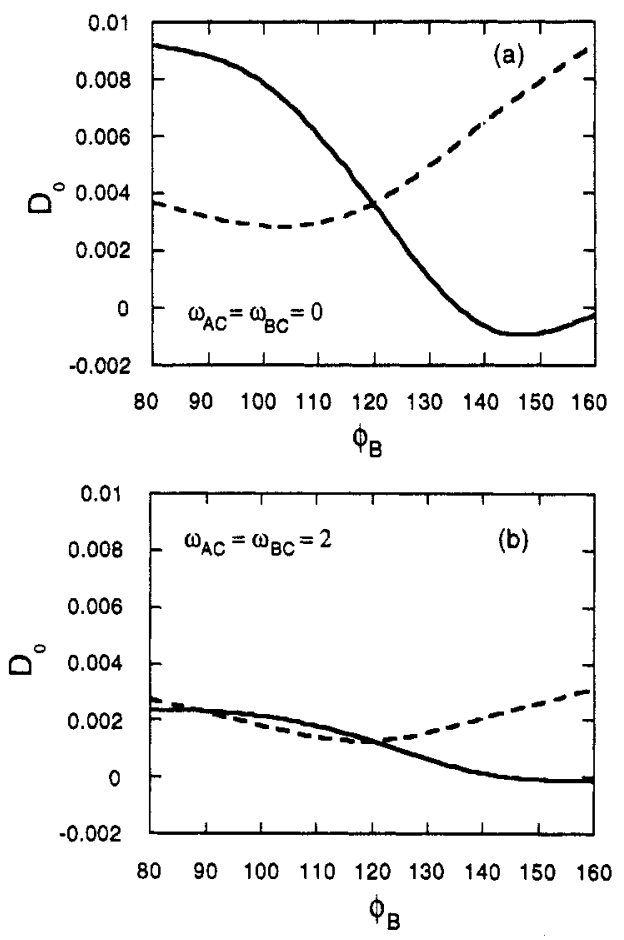

Figure 8. Effect of location of rotational isomeric states on the configurational factors $D_{0 \mathrm{~A}}$ and $D_{0 \mathrm{~B}}$ obtained for $u_{4}^{A}$ and $u_{\mathrm{H}}{ }^{\mathrm{B}}$ using the same parameters as in Figure 6 except for the secondorder interaction parameters (a) $\omega_{A C}=\omega_{B C}=0$ and (b) $\omega_{A C}=\omega_{B C}$ $=2$. See legend to Figure 6 .

The differences appearing in the values of $D_{0 \mathrm{~A}}$ and $D_{0 \mathrm{~B}}$ by $\mathrm{MC}$ chain generation result from the statistical insufficiency of the simulations.

Results of calculations for chains in which both units are assigned unfavorable second order interactions $\left(\omega_{\mathrm{AC}}\right.$ $\left.=\omega_{\mathrm{BC}}=0\right)$ and favorable interactions $\left(\omega_{\mathrm{AC}}=\omega_{\mathrm{BC}}=2\right)$ are shown in parts $a$ and $b$ of Figure 8, respectively. The curves are obtained for the orientations of $\mathbf{u}_{\|}{ }^{A}$ and $\mathbf{u}_{\|}{ }^{B}$. The orientations are strongly affected by the presence of unfavorable interactions as observed from Figure 8(a). In the opposite case of favorable interactions, units A and B exhibit comparable orientations as illustrated in Figure $8(b)$.

The orientations of $\mathbf{u}_{\perp}{ }^{A}$ and $\mathbf{u}_{\perp}{ }^{B}$ in the presence of second-order interactions are displayed in Figure 9. Irrespective of the type of second-order interactions, $D_{0 \mathrm{~A}}$ and $D_{0 \mathrm{~B}}$ are found to be indistinguishable for perpendicular vectors. The upper and lower curves in the figure are obtained for attractive $\left(\omega_{\mathrm{AC}}=\omega_{\mathrm{BC}}=2\right)$ and repulsive $\left(\omega_{\mathrm{AC}}\right.$ $=\omega_{B C}=0$ ) interactions, respectively. The middle dotted curve corresponds to the case of independent bonds. It is interesting to observe that in the presence of repulsive 


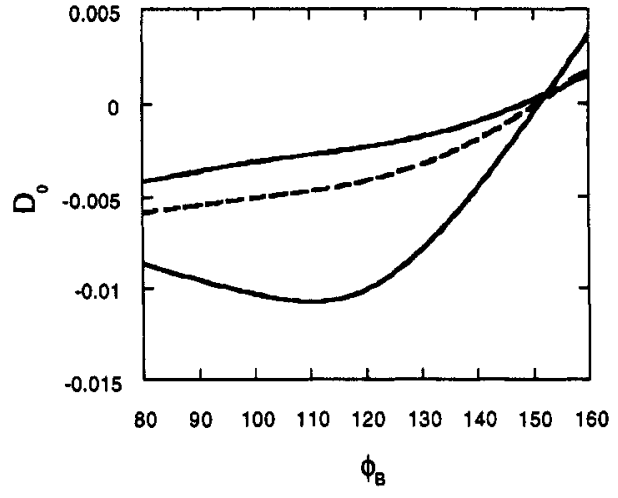

Figure 9. Effect of bond interdependence on $D_{0 \mathrm{~A}}$ and $D_{0 \mathrm{~B}}$ associated with $\mathbf{u}_{\perp}{ }^{A}$ and $\mathbf{u}_{\perp}{ }^{B}$. The upper and lower curves are obtained for attractive $\left(\omega_{A C}=\omega_{B C}=2\right)$ and repulsive $\left(\omega_{A C}=\omega_{B C}\right.$ $=0$ ) interactions, respectively. The middle dotted curve corresponds to the case of independent bonds $\left(\omega_{A C}=\omega_{B C}=1\right)$. The orientations of $\mathbf{u}_{\perp}{ }^{A}$ and $\mathbf{u}_{\perp}{ }^{B}$ are indistinguishable and represented by the same curve in each case.

interactions that preclude the $g^{ \pm} g^{\mp}$ states, segmental orientation changes drastically with the location of rotameric minima.

\section{Concluding Remark}

On the basis of the above analysis, we would like to emphasize the following major point: The intrinsic conformational properties of chain units exert a strong influence on segmental orientation. This feature leads to different degrees of orientation in different components of a copolymer, in particular for bond vectors. Even in the extreme case of an alternating copolymer, in which the orientational behavior of the adjacent monomeric units is expected to be closely coupled due to chain connectivity, significant deviations are observed in the orientations of vectors $u^{A}$ and $u^{B}$ appended to units $A$ and $B$, provided that the geometry and energy parameters of the two units are different. Thus, the fact that two units $A$ and $B$ belong to the same chain, being subject to the same extension ratio, does not necessarily imply the same orientation of different chain segments on a local scale.

Acknowledgment. Partial support by Bogazici University Research Fund Grant No. 93P0084 is gratefully acknowledged.

\section{References and Notes}

(1) Besbes, S.; Cermelli, I.; Bokobza, L.; Monnerie, L.; Bahar, I.; Erman, B.; Herz, J. Macromolecules 1992, 25, 1949.

(2) Dubault, A.; Deloche, B.; Herz, J. Macromolecules 1987, 20, 2096.

(3) Sotta, P.; Deloche, B. Macromolecules 1990, 23, 1999.

(4) Erman, B.; Mark, J. E. Annu. Rev. Phys. Chem. 1989, 40, 351.

(5) Flory, P. J. Statistical Mechanics of Chain Molecules; Interscience: New York, 1969. Flory, P. J. Macromolecules $1974,7,381$.

(6) Dubault, A.; Deloche, B.; Herz, J. Polymer 1984, 25, 1405.

(7) Deloche, B.; Dubault, A.; Herz, J.; Lapp, A. Europhys. Lett. $1986,1,629$.

(8) Bokobza, L.; Monnerie, L. Private correspondence.

(9) Besbes, S.; Bokobza, L.; Monnerie, L.; Bahar, I.; Erman, B. Polymer 1993, 34, 1179.

(10) Samuels, R. J. Structured Polymer Properties; Wiley: New York, 1974

(11) Ward, I. M. Structure and Properties of Oriented Polymers; Wiley: New York, 1975.

(12) Jasse, B.; Koenig, J. L. J. Macromol. Sci. Macromol. Chem. $1979, \mathrm{C} 17,61$.

(13) Jasse, B.; Tassin, J. F.; Monnerie, L. Prog. Colloid Polym. Sci. $1993,92,8$.

(14) Zhao, Y.; Jasse, B.; Monnerie, L. Polymer 1989, 30, 1643.

(15) Zhro, Y.; Jasse, B.; Monnerie, L. Polym. Commuñ. 1990, 31, 395.

(16) Zhao, Y.; Jasse, B.; Monnerie, L. Polymer 1991, 32, 209.

(17) Bouton, C.; Arrondel, V.; Rey, V.; Sergot, P.; Jasee, B.; Monnerie, L. Polymer 1989, 30, 1414.

(18) Faivre, J. P.; Jasse, B.; Monnerie, L. Polymer 1985, 26, 879.

(19) Nagai, K. J. Chem. Phys. 1964, 40, 2818.

(20) Erman, B.; Haliloglu, T.; Bahar, I.; Mark, J. E. Macromolecules $1991,24,901$.

(21) Fraser, R. D. B. J. Chem. Phys. 1953, 21, 1511.

(22) Read, B. E.; Stein, R. S. Macromolecules 1968, 1, 116.

(23) Bahar, I.; Erman, B. Macromolecules 1988, 21, 452. 